\title{
RNA sequencing reveals the circular RNA expression profiles of the infrapatellar fat pad/synovium unit
}

\author{
Tao Jiang ${ }^{1 \#}$, Yimin Lu ${ }^{1 \#}$, Zehua Chen ${ }^{1,2 \#}$, Xiaodong Lin $^{1}$, Jiayuan Zhang ${ }^{1,2}$, Jiaxin Shan ${ }^{1,2}$, Chao Lu ${ }^{1}$, \\ Chuanxi Zhao ${ }^{1}$, Xuemeng $\mathrm{Xu}^{1}$, Wengang $\mathrm{Liu}^{1}{ }^{\wedge}$ \\ ${ }^{1}$ Orthopedics Department, Guangdong Second Traditional Chinese Medicine Hospital, Guangzhou, China; ${ }^{2}$ The Fifth Clinical Medical College, \\ Guangzhou University of Traditional Chinese Medicine, Guangzhou, China \\ Contributions: (I) Conception and design: T Jiang; (II) Administrative support: W Liu, X Xu; (III) Provision of study materials or patients: W Liu, \\ C Zhao; (IV) Collection and assembly of data: Y Lu, J Shan; (V) Data analysis and interpretation: T Jiang, Z Chen; (VI) Manuscript writing: All \\ authors; (VII) Final approval of manuscript: All authors. \\ \#These authors contributed equally to this work. \\ Correspondence to: Wengang Liu; Xuemeng Xu. Orthopedics Department, Guangdong Second Traditional Chinese Medicine Hospital, Guangzhou \\ 510095, China. Email: 914194079@qq.com; xuxuemeng@163.com.
}

\begin{abstract}
Background: The infrapatellar fat pad (IPFP) and synovium are reported to act as a functional unit, with emerging roles in the pathophysiology of knee osteoarthritis (KOA). Circular RNAs (circRNAs) are involved in the pathogenesis of KOA, especially in cartilage homeostasis regulation. Nevertheless, the regulatory mechanisms of circRNAs in the KOA IPFP/synovium unit remain to be elucidated. Therefore, the current study aimed to investigate alterations in the expression of circRNAs and predict their functions in the KOA IPFP/synovium unit using bioinformatics analysis.
\end{abstract}

Methods: In brief, 6 synovium and IPFP specimens were collected, in which 3 from patients with KOA and 3 from controls. Then, circRNA sequencing was conducted on 2 KOA synovium and IPFP specimens as well as 1 control to investigate the expression profiles of circRNAs. Gene Ontology (GO), Kyoto Encyclopedia of Genes and Genomes (KEGG), and Reactome signaling pathway analyses were employed to predict the functions of the differentially expressed circRNAs. Based on the miRNA sponge theory, we constructed a circRNA-miRNA network to predict the molecular regulatory mechanism of these circRNAs. Venn analysis was performed to confirm the circRNAs and miRNAs expressed in both synovium and IPFP. Realtime quantitative reverse transcription-polymerase chain reaction (qRT-PCR) was also used to validate the expression levels of circRNAs that were co-expressed in both synovium and IPFP.

Results: A total of 65 and 72 circRNAs were differentially expressed in KOA synovium and IPFP, respectively (fold change $\geq 2, \mathrm{P}<0.05$ ). After obtaining the parental genes of differentially expressed circRNAs, the top 10 enrichment GO entries, KEGG pathways, and Reactome pathways were annotated. Furthermore, hsa_circ_0005265 was found to be down-regulated in both synovium and IPFP, which was validated by qRTPCR. The circRNA-miRNA network was created to annotate the probable regulatory mechanisms of the differentially expressed circRNAs, which consequently confirmed 2 target miRNAs (hsa-miR-6769b-5p and hsa-miR-1249-5p) associated with hsa_circ_0005265 in both synovium and IPFP.

Conclusions: Our outcomes bring us closer to understanding the potential mechanism of the IPFP/ synovium unit in the progression of KOA and finding new molecular targets for KOA therapy.

Keywords: Infrapatellar fat pad (IPFP); synovium; knee osteoarthritis (KOA); circular RNA (circRNA)

Submitted Sep 24, 2021. Accepted for publication Nov 22, 2021.

doi: $10.21037 / \mathrm{atm}-21-5739$

View this article at: https://dx.doi.org/10.21037/atm-21-5739

^ ORCID: Tao Jiang, 0000-0002-2837-6347; Yimin Lu, 0000-0002-5302-3615; Wengang Liu, 0000-0002-8887-8429. 


\section{Introduction}

Knee osteoarthritis (KOA) is one of the most common degenerative joint diseases and the main cause of joint pain and disability. The main causes leading to KOA included articular cartilage destruction, subchondral bone remodeling, synovitis, and osteophyte formation $(1,2)$. With the increasing aging population, KOA is expected to be the single greatest cause of disability in the general population by 2030 (3). Despite the increasing prevalence, the detailed molecular mechanism underlying the pathogenesis of KOA remains unknown. In recent years, it has been gradually recognized that KOA is a wholejoint disease (4). Researchers have begun to focus on the crosstalk (the adjacent anatomical location, highly similar pathological changes or composition of inflammatory cells, tight molecular communication) between the infrapatellar fat pad (IPFP) and synovium $(5,6)$. Moreover, several studies have demonstrated that the IPFP and synovium act as an anatomo-functional unit which plays a vital role in the inflammation cascade and cartilage degradation by secreting various cytokines, adipokines, fatty acids, and fatty acid derivatives (5-8). These studies suggest that the IPFP/ synovium unit may be a novel target for KOA.

As a type of non-coding RNA, circular RNAs (circRNAs) are widely exist in the cytoplasm of eukaryotic cells (9). CircRNAs are characterized by covalently closed-loop structures and high tissue-specific expression (10), and are thus involved in the regulation of transcriptional and posttranscriptional gene expression (11). Different from linear RNAs, circRNAs are more stable in vivo and can be stored in exosomes (12). Due to these characteristics, circRNAs have acted as potential diagnostic molecular biomarkers for various diseases such as Alzheimer's disease (13), rheumatoid arthritis (14), and atherosclerosis (15), among others. Recently, studies showed that circRNAs function as miRNA sponges that competitively suppress miRNA activity, which subsequently affects the downstream mRNA expression (16). To date, previous studies have only explored the link between circRNAs and KOA on cartilage (17-19) or synovium respectively (20), however, there is no study focusing on the circRNAs expression profile in the IPFP/ synovium unit.

In the present study, we reported differential expression profiles of circRNAs in KOA IPFP and synovium. Then, a series of systematic bioinformatics analyses were performed to clarify the circRNAs that are vital for the progression of KOA. Furthermore, an IPFP/synovium unit-related circRNA, hsa_circ_0005265, and its target miRNAs (hsa-miR-6769b-5p and hsa-miR-1249-5p) were identified in both IPFP and synovium for the first time. Thus, the current study intended to draw attention to the role of the IPFP/synovium unit in KOA development, and provide new insights into the pathogenesis of KOA. We present the following article in accordance with the MDAR reporting checklist (available at https://dx.doi. org/10.21037/atm-21-5739).

\section{Methods}

\section{IPFP and synovium samples}

IPFP and synovium were harvested from patients undergoing total knee arthroplasty (TKA) for end-stage KOA (KOA group, $\mathrm{n}=3$ ) or from patients undergoing anterior cruciate ligament reconstruction (ACLR) after traumatic rupture (normal control group, $n=3$ ) in Guangdong Second Traditional Chinese Medicine Hospital (Guangzhou, China). For each patient, small biopsies of IPFP and synovium were obtained during TKA or ACLR surgery. Then, tissues were stored in Dulbecco's modified Eagle's medium (DMEM) with 1\% bovine serum albumin (BSA) and quickly frozen in liquid nitrogen. The study was conducted in accordance with the Declaration of Helsinki (as revised in 2013). This study was approved by the Human Ethics Committee of Guangdong Second Traditional Chinese Medicine Hospital (approval code: 2020K56, approval date: 24 November 2020), and all participants signed informed consent.

\section{Total RNA extraction}

Total RNA from IPFP and synovium was isolated using Takara RNAiso Plus (total RNA extraction reagent) based on the manufacturer's instructions. The concentration and integrity of the extracted total RNA was estimated by the Qubit 3.0 Fluorometer (Invitrogen, Carlsbad, CA, USA) and Agilent 2100 Bioanalyzer (Applied Biosystems, Carlsbad, CA, USA), respectively. As a result, an RNA integrity number value of at least 7.0 or higher were used for further processing.

\section{Library preparation, investigation, clustering, and $\operatorname{circ} R N A$ sequencing}

Two $\mu \mathrm{g}$ of total RNA was used to construct the RNA-seq 
library by KAPA RNA HyperPrep Kit with RiboErase (HMR) for Illumina ${ }^{\circledR}$ (Kapa Biosystems, Inc., Woburn, MA, USA). In brief, ribosomal RNA was removed from the total RNA. The ribosome-depleted RNA was incubated for $30 \mathrm{~min}$ at $37^{\circ} \mathrm{C}$ with 10 units of RNase R (Epicentre Technologies, Madison, WI, USA) and purified with VAHTS RNA Clean Beads. Next, the ribominus RNase $\mathrm{R}(+)$ RNAs were fragmented and the first strand and directional second strand were synthesised. Then, the A tailing and adapter ligation were performed with the purified cDNA. Finally, the purified, adapter-ligated DNA was amplified. A DNA 1000 chip on an Agilent 2100 Bioanalyzer was used to assess the library quality and concentration. Accurate quantification for sequencing applications was determined using the qPCR-based KAPA Biosystems Library Quantification kit (Kapa Biosystems, Inc., Woburn, MA, USA). Each library was diluted to a final concentration of $10 \mathrm{nM}$ and pooled equimolar before clustering. The grouping of the index-coded samples was achieved on a cBot cluster generation system with TruSeq PE Cluster Kit v4-cBot-HS (Illumina) as per the supplier's protocol. Following the creation of the clusters, the libraries were sequenced on an Illumina Hiseq 4000 platform and $150 \mathrm{bp}$ paired-end reads were created.

\section{Differential circRNA expression analysis}

CIRI software (21) was used to recognize circRNAs. The expression of specific circRNAs was quantified by Spliced reads per billion mapping (SRPBM) (22). Then, the differentially expressed circRNAs were analyzed by DEGseq, which was suggested depending on an MA-plot. A $\mathrm{P}$ value was calculated for every circRNA. The differentially expressed circRNAs were selected using the following filter criteria: $\mathrm{P}$ value $<0.05$ and fold change $\geq 2$.

\section{Gene Ontology (GO), Kyoto Encyclopedia of Genes and Genomes (KEGG), and Reactome analyses}

The GO enrichment of differentially expressed circRNAs was analyzed by DAVID (Database for Annotation, Visualization, and Integrated Discovery) (23). The GO analysis was divided into 3 parts: biological process (BP), cellular component (CC), and molecular function (MF). The KEGG and Reactome enrichment analyses were performed to predict the related pathways of the parental genes of differentially expressed circRNAs. A P value of $<0.05$ reflected significant enrichment.

\section{Creation of the circRNA-miRNA interaction network}

Based on total-score and total-energy analyzed by miRanda and TargetScan (24), we predicted the circRNA-miRNA interaction networks and visualized it by using Cytoscape software.

\section{Venn analysis}

Venn analysis was conducted to identify the circRNAs and miRNAs expressed in both synovium and IPFP.

\section{Quantitative reverse transcription-polymerase chain reaction ( $q R T-P C R)$ analysis}

A circRNA (hsa_circ_0005265) co-differentially expressed in both IPFP and synovium was selected to verify its differential expression levels by qRTPCR. Briefly, cDNA was produced from total RNA by a Prime-Script RT reagent kit with gDNA Eraser (TaKaRa, Japan). The primers used for qRT-PCR are listed as follows: hsa_circ_0005265 (forward: 5'-CACTGAGTGGTAATGGATGATGC-3', reverse: 5'-GCTGAATGTTGGTGCCACAG-3'), GAPDH (forward: 5'-AAAGCCTGCCGGTGACTAAC-3', reverse: 5'-TTCCCGTTCTCAGCCTTGAC-3'). GAPDH acted as an internal control for the circRNA. All data are representative of 3 independent experiments performed in triplicate. The $2^{-\Delta \Delta \mathrm{Ct}}$ method was used to examine the relative expression levels of hsa_circ_0005265.

\section{Statistical analysis}

Data are presented as the mean \pm standard deviation (SD). Statistical significance was tested by Student's $t$-test, and a $\mathrm{P}$ value $<0.05$ was considered to be statistically significant. CircRNAs demonstrating fold changes of $\geq 2$ and $P$ values of $<0.05$ were regarded as significantly differentially expressed. All experiments were performed and analyzed in triplicate. Data analysis was performed with GraphPad Prism 7.0.

\section{Results}

\section{The circRNA expression profiles in KOA synovium and IPFP}

Differentially expressed circRNAs of KOA IPFP and synovium were displayed by heatmap and volcano plots after fold-change filtering (shown in Figure 1A-1D). 

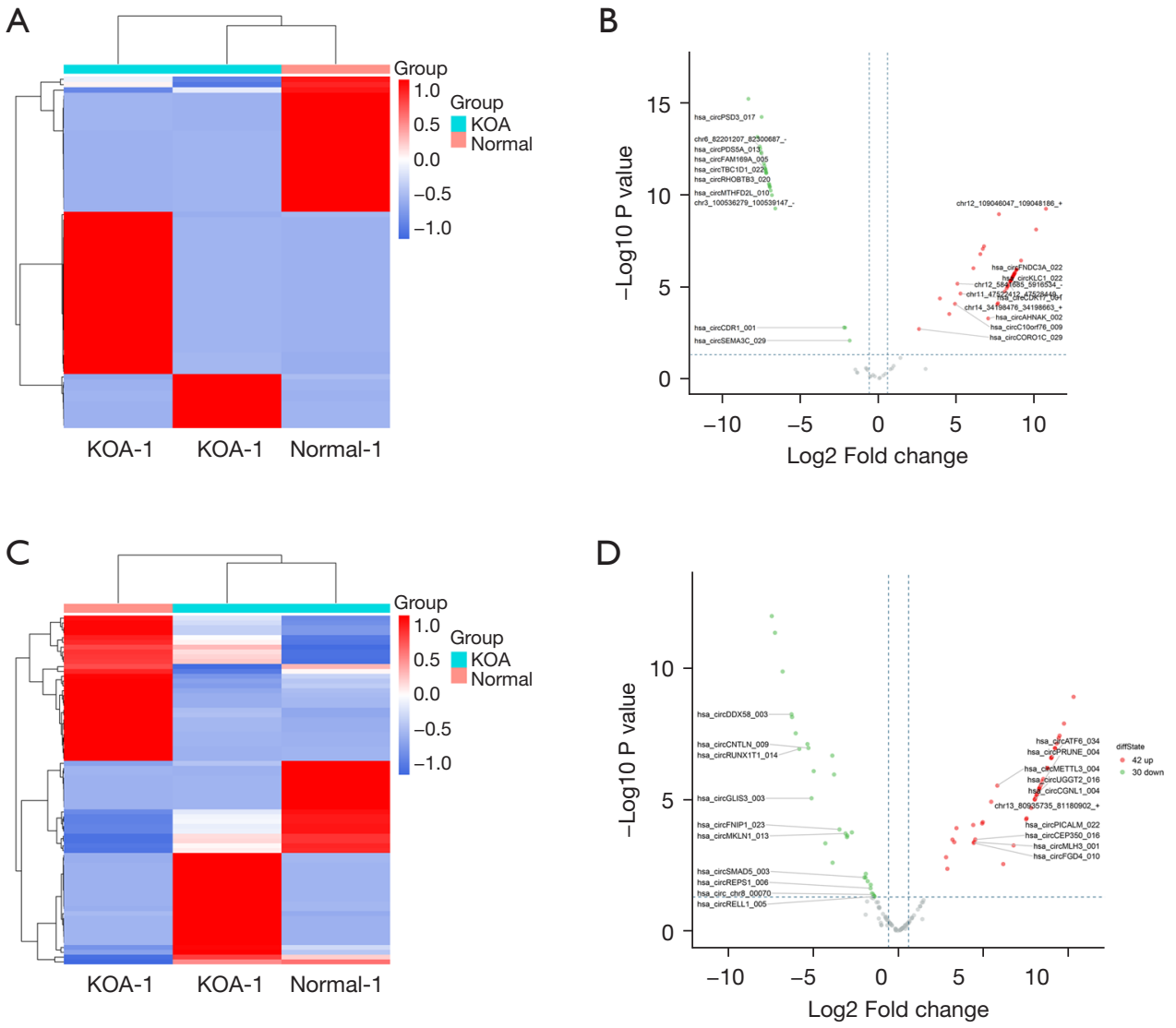

Figure 1 The circRNAs expression profiles between the KOA IPFP/synovium unit and normal controls. (A,C) Clustered heatmap of the differentially expressed circRNAs in KOA synovium and IPFP. Rows represent circRNAs while columns represent tissues. (B,D) Volcano plots were constructed based on fold change and $\mathrm{P}$ values. The red and green points represent the up-regulated or down-regulated circRNAs with statistical significance, respectively. circRNAs, circular RNAs; KOA, knee osteoarthritis; IPFP, infrapatellar fat pad.

In synovium, we identified a total of 65 circRNAs that were differentially expressed, including 40 up-regulated and 25 down-regulated circRNAs (Figure 1A,1B). According to the annotation of these circRNAs based on the RefSeq database, among the up-regulated circRNAs, 37 were transcribed from the exonic region, 1 from the intronic region, and 2 from the intergenic region, whereas the downregulated circRNAs consisted of 21 from the exonic region, 1 from the intronic region, 2 from the intergenic region, and 1 from other genomic regions. Overall, in KOA IPFP, 72 circRNAs were found to be significantly differentially expressed, comprising 42 up-regulated and 30 downregulated circRNAs (Figure 1C,1D). For the up-regulated circRNAs, 37 were transcribed from the exonic region, 3 from the intronic region, and 2 from the intergenic region. However, the down-regulated circRNAs consisted of 25 from the exonic region and 5 from the intronic region. Moreover, the top 10 up-regulated and down-regulated circRNAs in KOA synovium and IPFP are listed in Tables 1,2.

\section{Bioinformatics analysis of the parental genes of circRNAs}

To better understand the function of these differentially expressed circRNAs in KOA synovium and IPFP, we performed GO, KEGG, and Reactome analyses.

The GO analysis confirmed 51 significant GO terms (31 $\mathrm{BP}$ terms, $11 \mathrm{CC}$ terms, and $9 \mathrm{MF}$ terms) in synovium and 91 (62 BP terms, 8 CC terms, and $21 \mathrm{MF}$ terms) in IPFP. In terms of synovium, positive regulation of GTPase activity (GO: 0043547), regulation of DNA metabolic process (GO: 0051052), and regulation of GTPase activity (GO: 0043087) were the most enriched BP terms. In addition, other significant BP terms included B cell activation (GO: 0042113), positive regulation of DNA metabolic process 
Table 1 Top 10 up-regulated and down-regulated circRNAs in KOA synovium

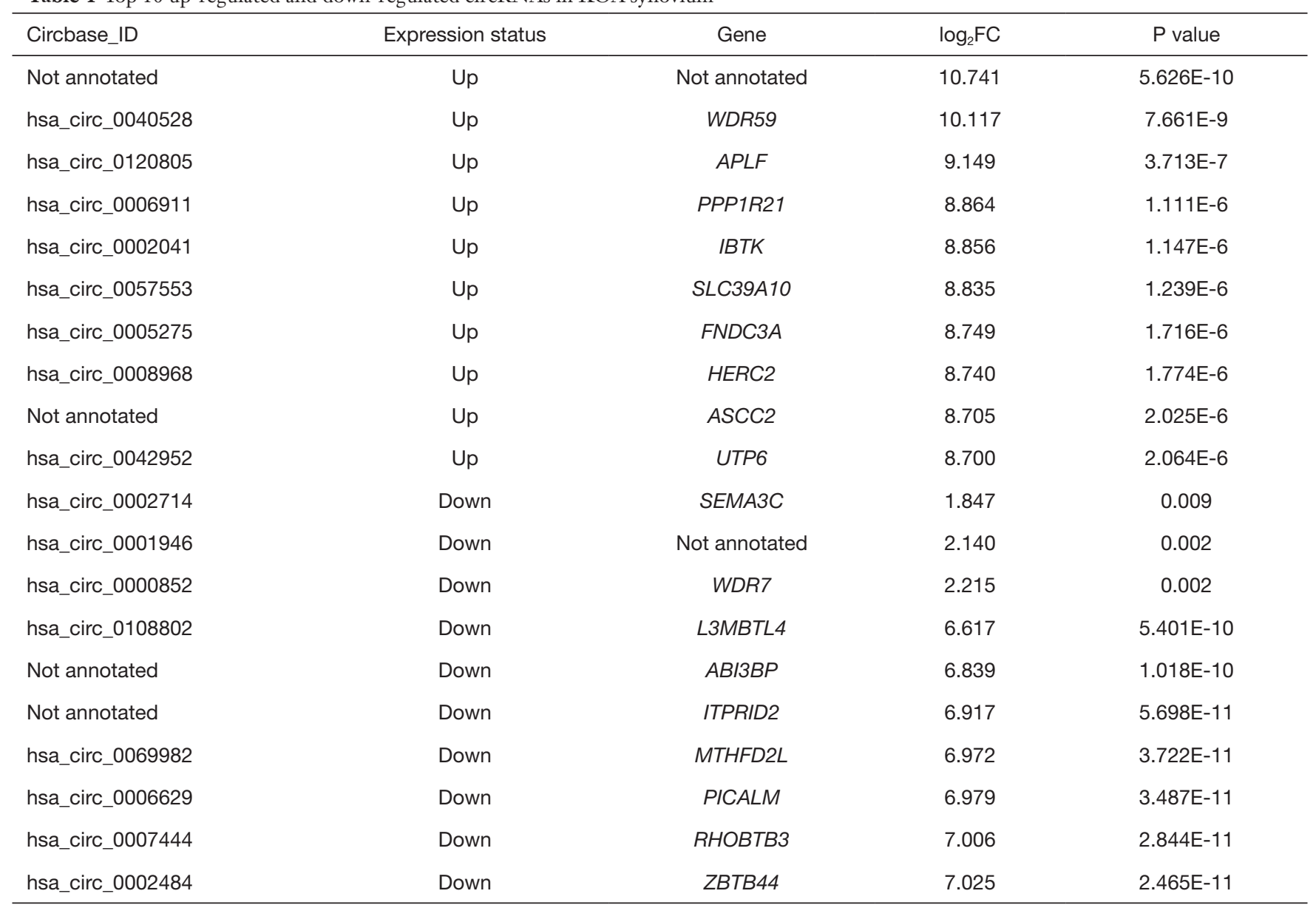

circRNA, circular RNA; KOA, knee osteoarthritis.

(GO: 0051054), signal transduction by 553 class mediator (GO: 0072331), and histone modification (GO: 0016570) (shown in https://cdn.amegroups.cn/static/public/ATM21-5739-1.xlsx). As for CC, the most enriched terms were chromosomal region (GO: 0098687) and chromosome, centromeric region (GO: 0000775). The most enriched MF terms were correlated with the regulation of GTPase (Figure $2 A$ ). In addition, for the IPFP, regulation of cell morphogenesis (GO: 0022604), microtubule cytoskeleton organization (GO: 0000226), and cell morphogenesis involved in neuron differentiation (GO: 0048667) were the most enriched BP terms. The most enriched CC terms were cell leading edge (GO: 0031252), cytoplasmic region (GO: 0099568), and actin cytoskeleton (GO: 0015629). Consistent with synovium, the most enriched MF terms were correlated with the regulation of GTPase (Figure 2B).

In the KEGG analysis, the top 10 pathways associated with the differentially expressed circRNAs of synovium and IPFP were listed (Figure 2C,2D). The most enriched KEGG terms related to synovium were influenza A (hsa05164) and the calcium signaling pathway (hsa04020). Furthermore, several other significant signaling pathways included the thyroid hormone signaling pathway (hsa04919), HIF-1 signaling pathway (hsa04066), and melanogenesis (hsa04916) (shown in https://cdn.amegroups.cn/ static/public/ATM-21-5739-1.xlsx). Correspondingly, transcriptional misregulation in cancer (hsa05202) and focal adhesion (hsa04510) were the most enriched KEGG terms of IPFP, in addition, other important KEGG terms included the ErbB signaling pathway (hsa04012), TGF- $\beta$ signaling pathway (hsa04350), and lysine degradation (hsa00310) (shown in https://cdn.amegroups.cn/static/public/ATM-215739-2.xlsx).

In addition to KEGG, Reactome is another well-known 
Table 2 Top 10 up-regulated and down-regulated circRNAs in KOA IPFP

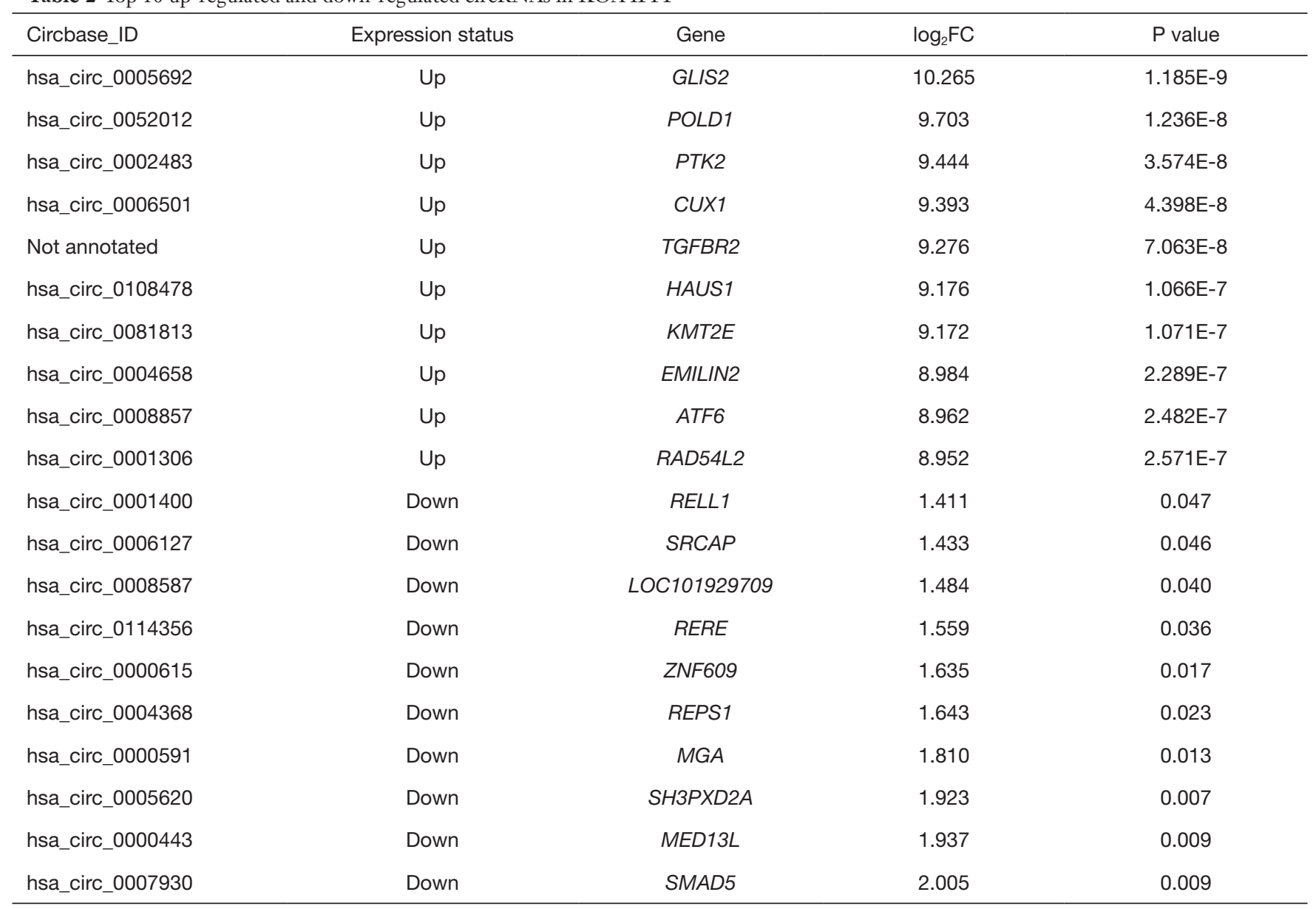

circRNA, circular RNA; KOA, knee osteoarthritis; IPFP, infrapatellar fat pad.

signaling pathway database. We also showed the top 10 pathways based on Reactome analysis in the present study (Figure $2 E, 2 F)$. The most enriched Reactome terms of synovium were cell cycle checkpoints (R-HSA-69620) and DNA repair (R-HSA-73894), while the most enriched Reactome term of IPFP was extracellular matrix (ECM) organization (R-HSA-1474244).

\section{Creation of the circRNA-miRNA interaction network}

CircRNAs are shown to act as miRNA sponges, thereby regulating the functions of relevant miRNAs via directly binding with miRNAs. According to total-score and totalenergy, we created the top 300 circRNA-miRNA networks of synovium and IPFP using miRanda, and mapped them using Cytoscape (Figure 3A,3B).

\section{Venn analysis}

Furthermore, we performed Venn analysis of circRNAs differentially expressed in synovium and IPFP, and screened for 1 circRNA named hsa_circ_0005265 that was downregulated in both synovium and IPFP (Figure 4). We also confirmed 2 target miRNAs (hsa-miR-6769b-5p and hsa-miR-1249-5p) related to hsa_circ_0005265 in both synovium and IPFP (Figure 5, Table 3).

\section{qRT-PCR validation of bsa_circ_0005265 in both synovium and IPFP}

Hsa_circ_0005265 was identified in both IPFP and synovium based on RNA sequencing, thus was selected for further qRT-PCR confirmation in 3 pairs of synovium and 
A

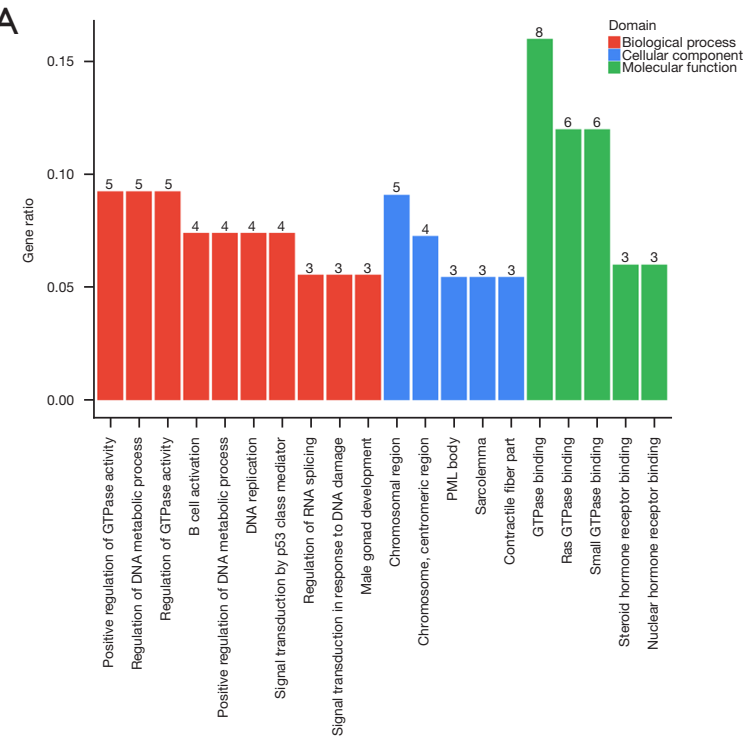

B

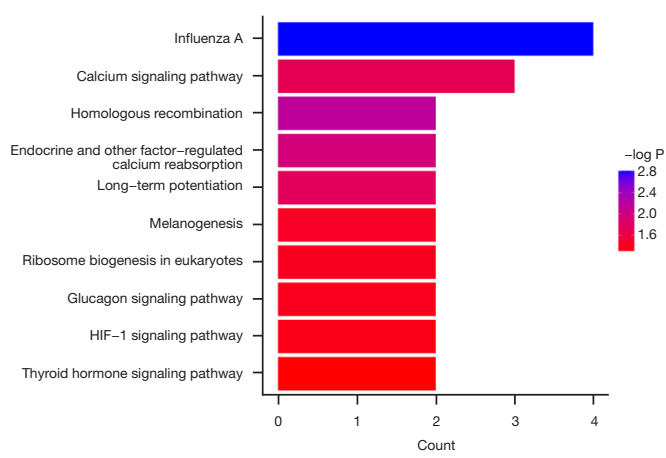

C

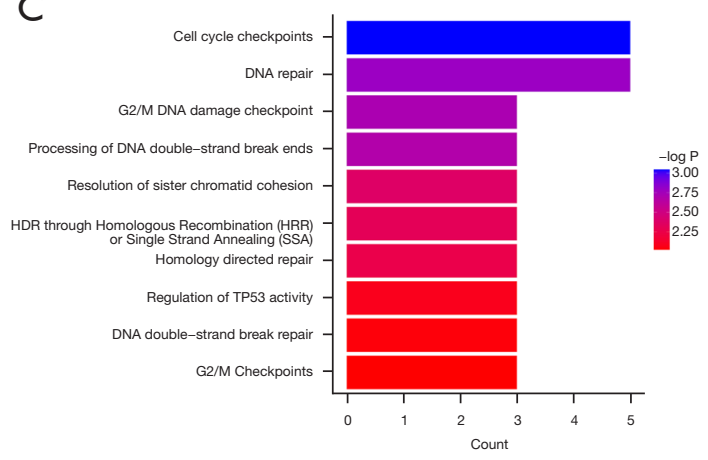

D

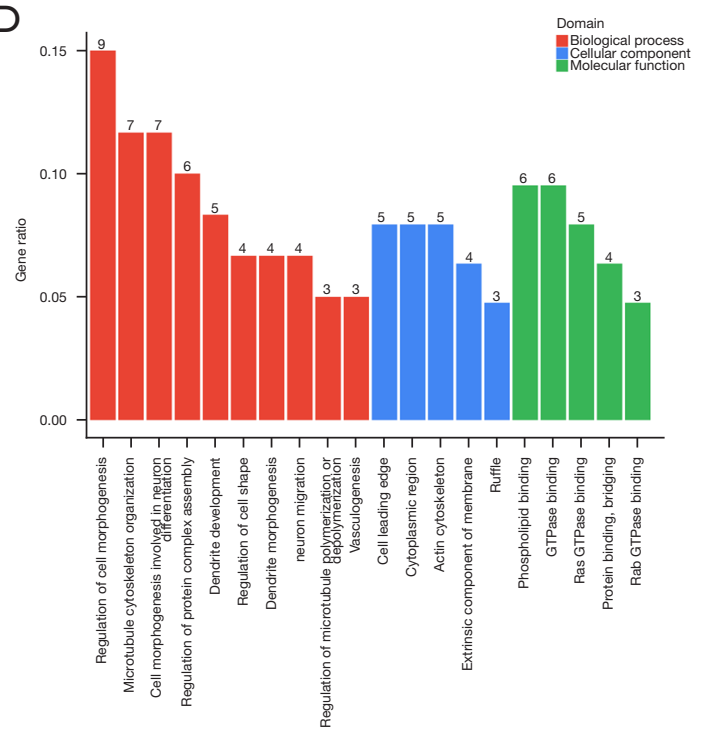

$\mathrm{E}$

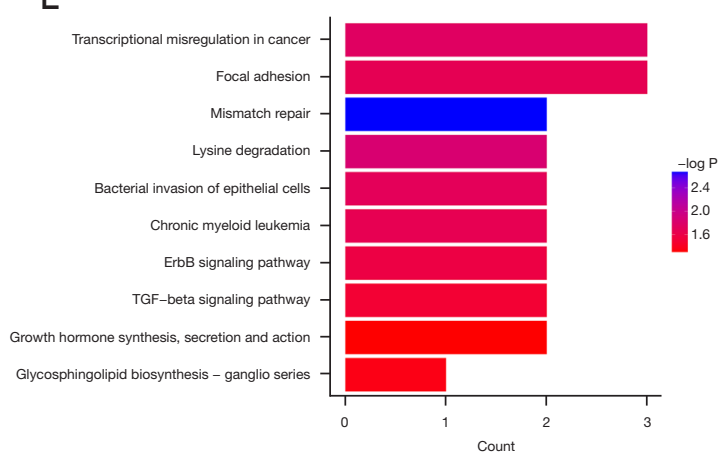

$\mathrm{F}$

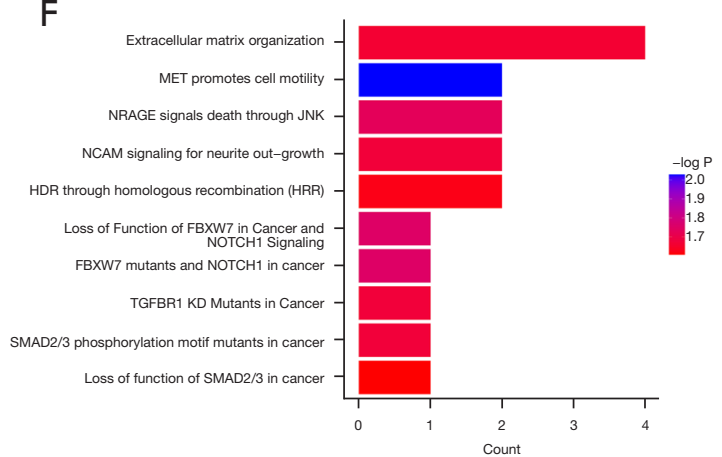

Figure 2 GO, KEGG, and Reactome analyses of the parental genes of the differentially expressed circRNAs in KOA synovium and IPFP. $(\mathrm{A}, \mathrm{B}) \mathrm{GO}$ analysis. BP, CC, MF are represented in red, blue, and green, respectively. (C,D) KEGG analysis. The top 10 KEGG pathways are shown. (E,F) Reactome analysis. The top 10 Reactome pathways are shown. GO, Gene Ontology; KEGG, Kyoto Encyclopedia of Genes and Genomes; KOA, knee osteoarthritis; IPFP, infrapatellar fat pad; BP, biological process; CC, cellular component; MF, molecular function; circRNA, circular RNA. 


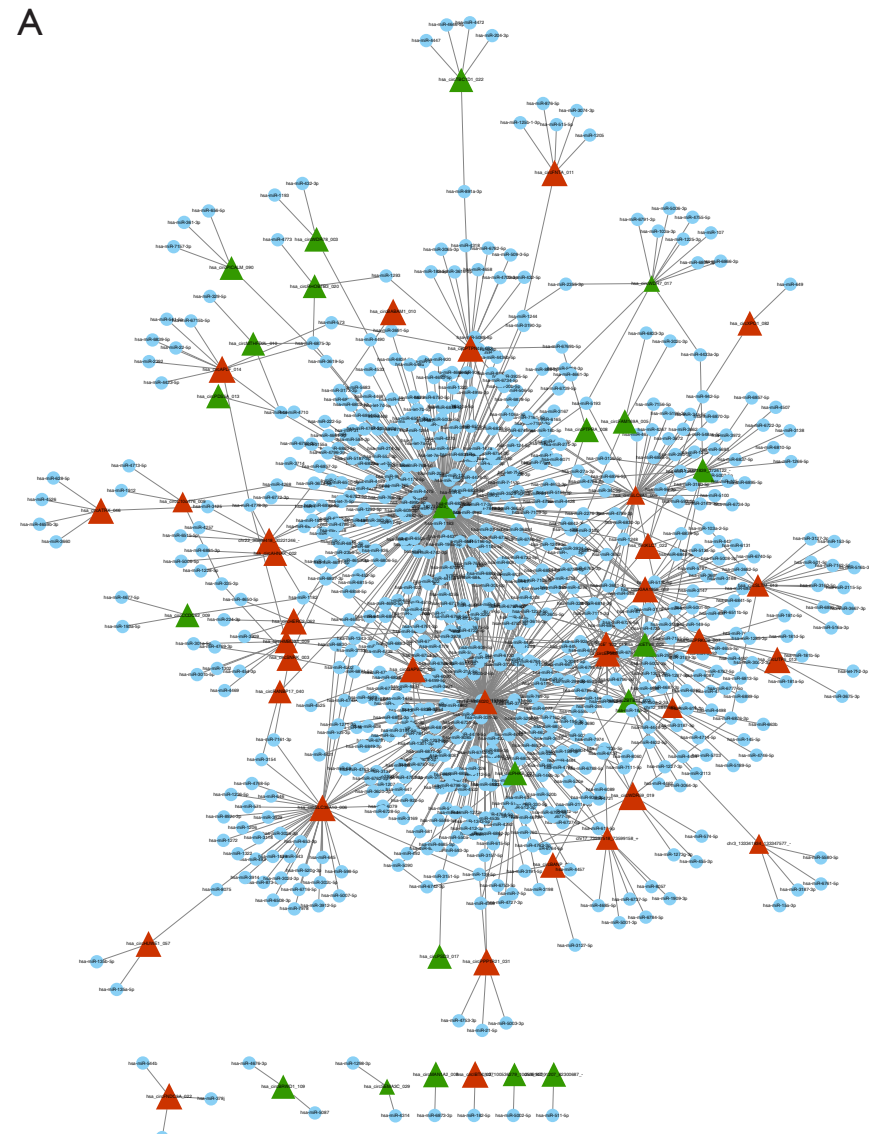

\section{B}

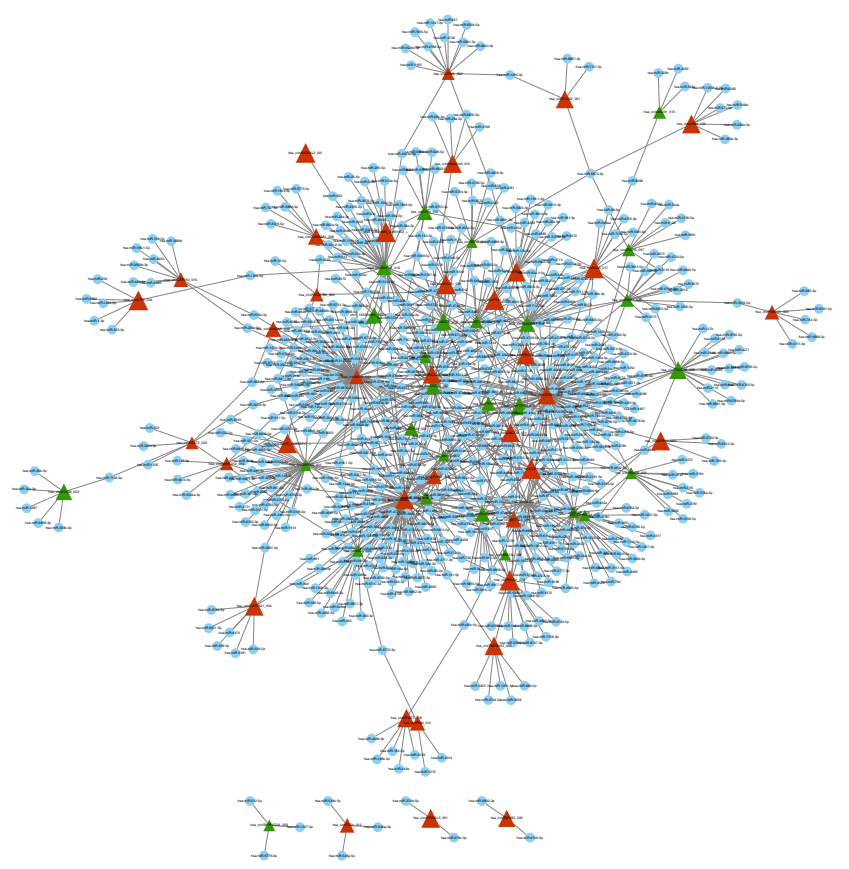

Figure 3 Co-expression network of differentially expressed circRNAs and miRNAs. Triangle nodes represent circRNAs and round nodes represent miRNAs. Red and green indicate up-regulated and down-regulated circRNAs, respectively. (A) CircRNA-miRNA networks of synovium. (B) CircRNA-miRNA networks of IPFP. IPFP, infrapatellar fat pad; circRNA, circular RNA.
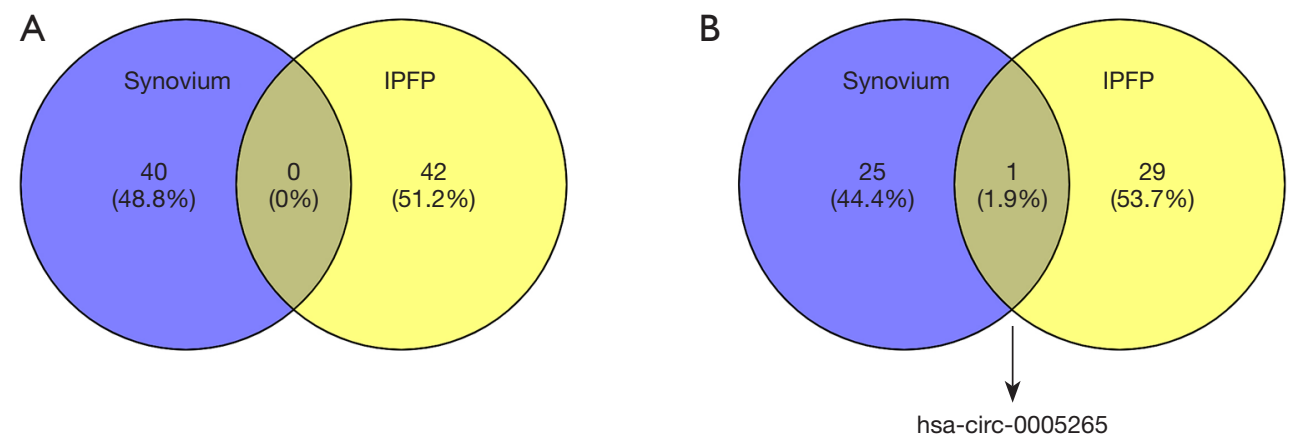

Figure 4 Venn analysis. (A) Up-regulated circRNAs in synovium and IPFP. (B) Down-regulated circRNAs in synovium and IPFP. IPFP, infrapatellar fat pad; circRNA, circular RNA.

IPFP samples. It was shown that the expression level of hsa_ circ_0005265 detected by qRT-PCR was highly consistent with the circRNA sequencing results, which demonstrated the high reliability of the RNA sequencing (Figure 6).

\section{Discussion}

$\mathrm{KOA}$ is one of the most prevalent chronic diseases. It is typically characterized by degradation of cartilage, though 

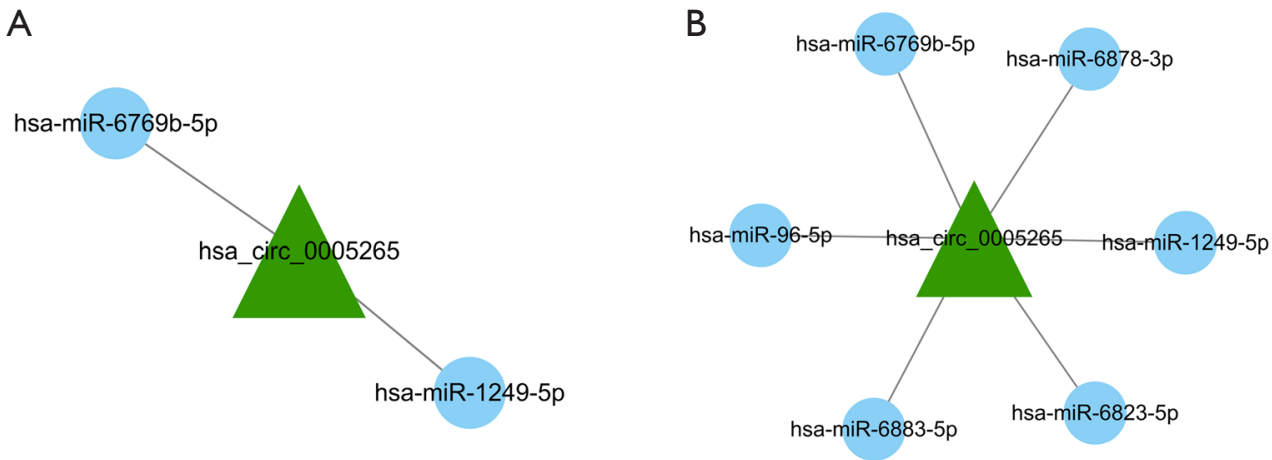

Figure 5 CircRNA-miRNA network of hsa_circ_0005265 in synovium and IPFP. (A) Target miRNAs of hsa_circ_0005265 in synovium. (B) Target miRNAs of hsa_circ_0005265 in IPFP. IPFP, infrapatellar fat pad; circRNA, circular RNA.

Table 3 Hsa_circ_0005265-related target miRNAs in synovium and IPFP

\begin{tabular}{lll}
\hline CircRNA ID & $\begin{array}{l}\text { Target miRNAs in } \\
\text { synovium }\end{array}$ & Target miRNAs in IPFP \\
\hline hsa_ & hsa-miR-6769b-5p & hsa-miR-6769b-5p \\
circ_0005265 & hsa-miR-1249-5p & hsa-miR-1249-5p \\
& hsa-miR-6823-5p \\
& hsa-miR-96-5p \\
& hsa-miR-6883-5 \\
& hsa-miR-6878-3p \\
\hline
\end{tabular}

CircRNA, circular RNA; IPFP, infrapatellar fat pad. the pathogenesis is not fully understood. Previous studies have tended to focus on the effects of single tissues such as cartilage, synovium, and IPFP on the development of KOA, while largely ignoring their synergistic effects. In fact, crosstalk was found between cartilage and other tissues within the knee joint $(25,26)$. Over the last decade, KOA has been considered as a whole-joint disease $(4,5)$, as cartilage, subchondral bone, meniscus, IPFP, and synovium, among others, are involved in the progression of KOA. Among these tissues, IPFP and synovium are regarded as a morphological and functional unit $(7,8)$ based on their adjacent anatomical location, highly similar pathological changes or composition of inflammatory cells, and tight

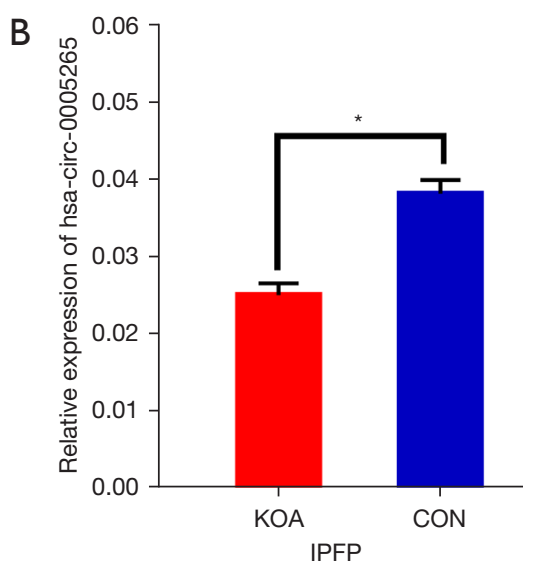

Figure 6 Relative expression level of hsa_circ_0005265 detected by qRT-PCR in synovium and IPFP. The qRT-PCR results were consistent with the RNA sequencing results. * $\mathrm{P}<0.05$. KOA, knee osteoarthritis; CON, control; qRT-PCR, quantitative reverse transcriptionpolymerase chain reaction; IPFP, infrapatellar fat pad. 
molecular crosstalk, among other factors $(4,5,27,28)$. Studies indicated that the typical changes of the IPFP/ synovium unit in the progression of $\mathrm{KOA}$ include fibrosis, vascularization, and inflammatory infiltration. Furthermore, it can secrete adipokines, pro-inflammatory factors, such as IL- $1 \beta$ and TNF- $\alpha$, cartilage matrix-degrading enzymes, such as MMP-13 and ADAMTS-5, and fatty acids and their derivatives, thus mediating cartilage degeneration $(4,7,26,29)$. However, the underlying mechanism by which the IPFP/synovium unit regulates this process remains unclear.

Along with the continuous development of sequencing technologies and bioinformatics technology, circRNAs are emerging as a research hotspot in KOA. CircRNAs are reported to regulate gene expression via diverse mechanisms. The most widely studied regulatory mechanism is their role as miRNAs sponge, which implies that they can interact with miRNAs and repress their functions, consequently regulating mRNA transcription and protein production (18-20). In recent years, there has been an increasing amount of studies concerned with the association between circRNAs and KOA (17-19). Shen et al. explored the expression profiles of circRNAs in OA cartilage, and found circSERPINE2 was down-regulated in the OA cartilage tissues. Further research demonstrated that the function of circSERPINE2, as a sponge of miR-1271$5 \mathrm{p}$, was related to excessive apoptosis and the imbalance between anabolic and catabolic factors of the ECM (17). In addition, Zhou et al. showed that circRNA_33186 was up-regulated in both IL-1 $\beta$-treated chondrocytes and cartilage tissues of a destabilized medial meniscus (DMM)induced KOA mouse model. Then, the mechanistic study found that circRNA_33186 directly binds to miR-127-5p, thereby inhibiting miR-127-5p and increasing MMP-13 expression, thus contributing to KOA pathogenesis (19). Other similar research has proven the protective role of circPDE4D, which exerted its effect by acting as a sponge for $\mathrm{miR}-103 \mathrm{a}-3 \mathrm{p}$ and thereby playing a critical role in maintaining the ECM during KOA progression (18). These studies highlight the regulatory roles of circRNAs in the development of KOA, especially in cartilage homeostasis, and provide a potentially effective therapeutic strategy for KOA progression.

CircRNAs are abundantly expressed in synovium and adipose tissues. To date, no research has highlighted the expression profiles of circRNAs in the IPFP/synovium unit. Taking this into account, it may be possible to reveal the role of the IPFP/synovium unit in the pathogenesis of KOA. In the present study, we aimed to clarify the circRNA regulation in the IPFP/synovium unit and screen for circRNAs that were differentially expressed in both synovium and IPFP. As a result, for the samples from KOA patients, we detected 65 circRNAs and 72 circRNAs that were differentially expressed in synovium and IPFP, respectively. Then, GO, KEGG, and Reactome analyses were conducted to functionally annotate parental genes of differentially expressed circRNAs.

For synovium, the parental genes were functionally related to cell metabolism, immune modulation, inflammation regulation, and histone modification, as well as being enriched in several pathways such as the calcium signaling pathway, thyroid hormone signaling pathway, HIF-1 signaling pathway, and melanogenesis. Mechanical loading is one of the most important external factors regulating cartilage metabolism, and calcium signaling is reported as an initial step in chondrocyte mechanotransduction (30), which is an essential component in differentiating and mature chondrocytes (31). Thyroid hormone has been implicated in the normal growth and development of articular cartilage. Bundy et al. found that compared to normal controls, skeletally immature miniature swine induced to a hypothyroid state showed remarkably increased mean articular cartilage thickness and decreased mean chondrocyte density (32). The effects of HIF-1 $\alpha$ in KOA have also been explored for decades (33). Briefly, the hypoxic microenvironment could induce endoplasmic reticulum stress in chondrocytes and further disturb ECM secretion (34,35). Anti-oxidative agents exhibit great potential in KOA therapy. Zhong et al. indicated that dopamine melanin nanoparticles function as novel scavengers of reactive oxygen species (ROS) and reactive nitrogen species (RNS), which in turn slowed down cartilage degradation (36).

As for IPFP, the parental genes were related to cell metabolism, cell morphogenesis regulation, cell differentiation, and bone development. Besides, according to the results, the enriched signaling pathways including the ErbB signaling pathway, TGF- $\beta$ signaling pathway, and lysine degradation. The ErbB signaling pathway has been rarely studied in KOA, though Fisher et al. identified the positive role of endogenous ErbB signaling in chondrocyte proliferation (37). TGF- $\beta$ signaling is involved in the pathogenesis of KOA, as TGF- $\beta$ signaling inhibition in mesenchymal stem cells (MSCs) of the subchondral bone attenuates KOA (38), whereas activating TGF- $\beta$ signaling accelerates cartilage injury (39). During the past decade, 
tissue engineering has shown promising clinical application prospects in cartilage repair. Poly L-lysine (PLL), a polypeptide with cationic lysine groups as its functional group, has been shown to enhance chondrogenesis in a dose and molecular weight dependent manner in vitro (40). However, the underlying mechanisms of circRNAs in these signaling pathways in KOA remain unclear, which need further study.

Additionally, hsa_circ_0005265 was found to be downregulated in both synovium and IPFP through Venn analysis, and was verified using qRT-PCR in $3 \mathrm{KOA}$ tissues and 3 normal tissues. Then, we explored the potential regulatory mechanism of hsa_circ_0005265. Based on the aforementioned miRNA sponge theory, a circRNA-miRNA interaction network was created in the current study. The predicted miRNAs were selected according to total score and total energy using miRanda, and the smaller the total energy or the larger the total score, the closer the combination between the circRNAs and the miRNAs. It was found that hsa_circ_0005265 potentially interacted with 2 miRNAs in synovium and 6 miRNAs in IPFP. Of note, both hsa-miR-6769b-5p and hsa-miR-1249-5p existed in the 2 networks, suggested that hsa_circ_0005265 may regulate these 2 miRNAs and subsequently affects the downstream mRNA expression, thus play a vital role in the pathophysiology of KOA. Among these miRNAs, 5 have already been studied. The up-regulation of miR-6769b-5p in bone marrow-derived macrophages (BMDMs) led to a fibrolytic phenotype, where MMP-9 was up-regulated while inflammatory mediator expression was decreased by the suppression of ATF4 expression, which suggested that the miR-6769b$5 \mathrm{p} / \mathrm{ATF} 4$ axis may be a potential therapeutic target for chronic liver disease (41). MiR-1249-5p was involved in the regulation of bone metabolism $(42,43)$, which can positively regulate osteogenic differentiation of adiposederived stem cells via targeting PDX1 through the PI3K/ Akt signaling pathway (43). Exosomes are small, singlemembrane, secreted vesicles which play essential roles in intercellular communications and transport a variety of intracellular components, including miRNAs. For example, exosomes-derived miR-6823-5p has been shown in effective targeting for pancreatic cancer treatment (44). Osteosarcoma (OS) is a malignant tumor that tends to occur in adolescents. MiRNAs have been found to be abnormally expressed in OS tissue and act as potential targets for the prognosis and treatment of OS. A study indicated that the expression level of miR-96-5p was increased in OS cell lines and tissues. In addition, miR-96$5 \mathrm{p}$ mimic significantly inhibited proliferation and invasion while promoting the apoptosis of OS cells, implying that it may function as an effective target in OS treatment (45). CDK4/6 targeting is a promising therapeutic strategy for various types of neoplasms. CDK4/6 targeting by miR6883-5p exhibited anti-proliferative effects in human colorectal cancer cells (46). However, the association between these miRNAs and the pathogenesis of KOA remains unknown. It is necessary to carry out further studies to clarify the potential mechanisms of these miRNAs in the occurrence and development of KOA. Moreover, no study has focused on the role of miR-6878$3 \mathrm{p}$ in basic medicine.

This is the first study to examine the circRNA profiles in the IPFP/synovium unit by RNA sequencing and bioinformatics analysis. However, the detailed molecular mechanism of circRNAs in KOA pathogenesis need further exploration. Of note, there are some limitations in the present study. Firstly, based on the sponge theory, circRNAs may have sufficient miRNA binding sites, and further validation of the interaction between targeted miRNAs and the differentially expressed circRNAs might help elucidate the role of the IPFP/synovium unit in KOA. Secondly, in addition to the common sponge mechanism, other possible mechanisms such as regulating transcription or translation into proteins associated with KOA remain unclear. Further studies are necessary to explore the potential roles of the IPFP/synovium unit associated circRNAs such as hsa circ_0005265 in the pathogenesis of KOA.

\section{Conclusions}

In summary, the IPFP/synovium unit is involved in the pathogenesis of KOA. Our study identified the circRNA profiles in the KOA IPFP/synovium unit for the first time. GO, KEGG, and Reactome signaling pathway analyses were performed to annotate the potential functions of the differentially expressed circRNAs. Furthermore, to elucidate the regulatory mechanism, the circRNA-miRNA interaction network was created using bioinformatics methods. More importantly, our research found that hsa circ_0005265 was down-regulated in both synovium and IPFP, and predicted 2 common target miRNAs (hsamiR-6769b-5p and hsa-miR-1249-5p) related to hsa circ_0005265 in these 2 tissues. However, intensive study is needed to explain the molecular mechanisms of circRNAs in the KOA IPFP/synovium unit. We hope this study draws 
more attention to the IPFP/synovium unit, which may improve our understanding of the pathogenesis of KOA and help offer new insights for the clinical treatment of KOA.

\section{Acknowledgments}

Funding: This work was supported by Science and Technology Planning Project of Guangzhou City of China (Grant Number: 202002030204), Medical Science and Technology Research Fund Project of Guangdong Province of China (Grant Number: A2021463), Natural Science Foundation of Guangdong Province of China (Grant Number: 2021A1515011545), and Science and Technology Planning Project of Guangdong Province of China (Grant Number: 2021B1111610007).

\section{Footnote}

Reporting Checklist: The authors have completed the MDAR reporting checklist. Available at https://dx.doi. org/10.21037/atm-21-5739

Data Sharing Statement: Available at https://dx.doi. org/10.21037/atm-21-5739

Conflicts of Interest: All authors have completed the ICMJE uniform disclosure form (available at https://dx.doi. org/10.21037/atm-21-5739). XX, ZC and TJ report funding from Natural Science Foundation of Guangdong Province of China (Grant Number: 2021A1515011545) and from Science and Technology Planning Project of Guangdong Province of China (Grant Number: 2021B1111610007). WL and TJ report funding from Science and Technology Planning Project of Guangzhou City of China (Grant Number: 202002030204), and from Medical Science and Technology Research Fund Project of Guangdong Province of China (Grant Number: A2021463). The other authors have no conflicts of interest to declare.

Ethical Statement: The authors are accountable for all aspects of the work in ensuring that questions related to the accuracy or integrity of any part of the work are appropriately investigated and resolved. The study was conducted in accordance with the Declaration of Helsinki (as revised in 2013). This study was approved by the Human Ethics Committee of Guangdong Second Traditional Chinese Medicine Hospital (approval code: 2020K56, approval date: 24 November 2020), and all participants signed informed consent.

Open Access Statement: This is an Open Access article distributed in accordance with the Creative Commons Attribution-NonCommercial-NoDerivs 4.0 International License (CC BY-NC-ND 4.0), which permits the noncommercial replication and distribution of the article with the strict proviso that no changes or edits are made and the original work is properly cited (including links to both the formal publication through the relevant DOI and the license). See: https://creativecommons.org/licenses/by-nc-nd/4.0/.

\section{References}

1. Choi WS, Lee G, Song WH, et al. The CH25HCYP7B1-ROR $\alpha$ axis of cholesterol metabolism regulates osteoarthritis. Nature 2019;566:254-8.

2. Bennell KL, Hunter DJ. Physical Therapy before the Needle for Osteoarthritis of the Knee. N Engl J Med 2020;382:1470-1.

3. Thomas E, Peat G, Croft P. Defining and mapping the person with osteoarthritis for population studies and public health. Rheumatology (Oxford) 2014;53:338-45.

4. Eymard F, Pigenet A, Citadelle D, et al. Knee and hip intra-articular adipose tissues (IAATs) compared with autologous subcutaneous adipose tissue: a specific phenotype for a central player in osteoarthritis. Ann Rheum Dis 2017;76:1142-8.

5. Klein-Wieringa IR, de Lange-Brokaar BJ, Yusuf E, et al. Inflammatory Cells in Patients with Endstage Knee Osteoarthritis: A Comparison between the Synovium and the Infrapatellar Fat Pad. J Rheumatol 2016;43:771-8.

6. Favero M, El-Hadi H, Belluzzi E, et al. Infrapatellar fat pad features in osteoarthritis: a histopathological and molecular study. Rheumatology (Oxford) 2017;56:1784-93.

7. Macchi V, Stocco E, Stecco C, et al. The infrapatellar fat pad and the synovial membrane: an anatomo-functional unit. J Anat 2018;233:146-54.

8. Greif DN, Kouroupis D, Murdock CJ, et al. Infrapatellar Fat Pad/Synovium Complex in Early-Stage Knee Osteoarthritis: Potential New Target and Source of Therapeutic Mesenchymal Stem/Stromal Cells. Front Bioeng Biotechnol 2020;8:860.

9. Conn SJ, Pillman KA, Toubia J, et al. The RNA binding protein quaking regulates formation of circRNAs. Cell 2015;160:1125-34.

10. Memczak S, Jens M, Elefsinioti A, et al. Circular RNAs are a large class of animal RNAs with regulatory potency. 
Nature 2013;495:333-8.

11. Salzman J, Gawad C, Wang PL, et al. Circular RNAs are the predominant transcript isoform from hundreds of human genes in diverse cell types. PLoS One 2012;7:e30733.

12. Li Y, Zheng Q, Bao C, et al. Circular RNA is enriched and stable in exosomes: a promising biomarker for cancer diagnosis. Cell Res 2015;25:981-4.

13. Lukiw WJ. Circular RNA (circRNA) in Alzheimer's disease (AD). Front Genet 2013;4:307.

14. Zheng F, Yu X, Huang J, et al. Circular RNA expression profiles of peripheral blood mononuclear cells in rheumatoid arthritis patients, based on microarray chip technology. Mol Med Rep 2017;16:8029-36.

15. Zhao Z, Li X, Gao C, et al. Peripheral blood circular RNA hsa_circ_0124644 can be used as a diagnostic biomarker of coronary artery disease. Sci Rep 2017;7:39918.

16. Hansen TB, Jensen TI, Clausen BH, et al. Natural RNA circles function as efficient microRNA sponges. Nature 2013;495:384-8.

17. Shen $\mathrm{S}, \mathrm{Wu} \mathrm{Y}$, Chen J, et al. CircSERPINE2 protects against osteoarthritis by targeting miR-1271 and ETSrelated gene. Ann Rheum Dis 2019;78:826-36.

18. Wu Y, Hong Z, Xu W, et al. Circular RNA circPDE4D Protects against Osteoarthritis by Binding to miR-103a-3p and Regulating FGF18. Mol Ther 2021;29:308-23.

19. Zhou ZB, Huang GX, Fu Q, et al. circRNA.33186 Contributes to the Pathogenesis of Osteoarthritis by Sponging miR-127-5p. Mol Ther 2019;27:531-41.

20. Xiang S, Li Z, Bian Y, et al. RNA sequencing reveals the circular RNA expression profiles of osteoarthritic synovium. J Cell Biochem 2019;120:18031-40.

21. Gao Y, Wang J, Zhao F. CIRI: an efficient and unbiased algorithm for de novo circular RNA identification. Genome Biol 2015;16:4.

22. Liu X, Shi X, Chen C, et al. Improving RNA-Seq expression estimation by modeling isoform- and exonspecific read sequencing rate. BMC Bioinformatics 2015;16:332.

23. Huang da W, Sherman BT, Lempicki RA. Systematic and integrative analysis of large gene lists using DAVID bioinformatics resources. Nat Protoc 2009;4:44-57.

24. Agarwal V, Bell GW, Nam JW, et al. Predicting effective microRNA target sites in mammalian mRNAs. Elife 2015;4:e05005.

25. Hu W, Chen Y, Dou C, et al. Microenvironment in subchondral bone: predominant regulator for the treatment of osteoarthritis. Ann Rheum Dis 2020;80:413-22.
26. Zeng N, Yan ZP, Chen XY, et al. Infrapatellar Fat Pad and Knee Osteoarthritis. Aging Dis 2020;11:1317-28.

27. Bastiaansen-Jenniskens YM, Wei W, Feijt C, et al. Stimulation of fibrotic processes by the infrapatellar fat pad in cultured synoviocytes from patients with osteoarthritis: a possible role for prostaglandin $\mathrm{f} 2 \alpha$. Arthritis Rheum 2013;65:2070-80.

28. Eymard F, Pigenet A, Citadelle D, et al. Induction of an inflammatory and prodegradative phenotype in autologous fibroblast-like synoviocytes by the infrapatellar fat pad from patients with knee osteoarthritis. Arthritis Rheumatol 2014;66:2165-74.

29. Ioan-Facsinay A, Kloppenburg M. Osteoarthritis: Inflammation and fibrosis in adipose tissue of osteoarthritic joints. Nat Rev Rheumatol 2017;13:325-6.

30. Ogawa H, Akiyama H. Cartilage/chondrocyte research and osteoarthritis. Mechanobiology for development of osteoarthritis. Clin Calcium 2018;28:789-95.

31. Madden RM, Han SK, Herzog W. The effect of compressive loading magnitude on in situ chondrocyte calcium signaling. Biomech Model Mechanobiol 2015;14:135-42.

32. Bundy JA, Yang JT, Morscher MA, et al. Induced hypothyroidism alters articular cartilage in skeletally immature miniature swine. Connect Tissue Res 2020. doi: 10.1080/03008207.2020.1839436. [Epub ahead of print].

33. Stegen S, Laperre K, Eelen G, et al. HIF-1 $\alpha$ metabolically controls collagen synthesis and modification in chondrocytes. Nature 2019;565:511-5.

34. Fernández-Torres J, Martínez-Nava GA, Zamudio-Cuevas $\mathrm{Y}$, et al. Impact of the gene-gene interactions related to the HIF-1 $\alpha$ signaling pathway with the knee osteoarthritis development. Clin Rheumatol 2019;38:2897-907.

35. Yudoh K, Nakamura H, Masuko-Hongo K, et al. Catabolic stress induces expression of hypoxia-inducible factor (HIF)-1 alpha in articular chondrocytes: involvement of HIF-1 alpha in the pathogenesis of osteoarthritis. Arthritis Res Ther 2005;7:R904-R914. Erratum in Arthritis Res Ther 2005;7:225.

36. Zhong G, Yang X, Jiang X, et al. Dopamine-melanin nanoparticles scavenge reactive oxygen and nitrogen species and activate autophagy for osteoarthritis therapy. Nanoscale 2019;11:11605-16.

37. Fisher MC, Clinton GM, Maihle NJ, et al. Requirement for ErbB2/ErbB signaling in developing cartilage and bone. Dev Growth Differ 2007;49:503-13.

38. Zhen $\mathrm{G}$, Wen $\mathrm{C}$, Jia $\mathrm{X}$, et al. Inhibition of TGF- $\beta$ signaling in mesenchymal stem cells of subchondral bone 
Page 14 of 14

attenuates osteoarthritis. Nat Med 2013;19:704-12.

39. Wang YJ, Shen M, Wang S, et al. Inhibition of the TGF- $\beta 1 /$ Smad signaling pathway protects against cartilage injury and osteoarthritis in a rat model. Life Sci 2017;189:106-13.

40. Kim YS, Chien AJ, Guo JL, et al. Chondrogenesis of cocultures of mesenchymal stem cells and articular chondrocytes in poly(l-lysine)-loaded hydrogels. J Control Release 2020;328:710-21.

41. Nishi M, Matsumoto T, Fujisawa K, et al. Mesenchymal Stem Cells Induce a Fibrolytic Phenotype By Regulating mmu-miR-6769b-5p Expression in Macrophages. Stem Cells Dev 2020;29:1457-66.

42. Wu J, Ren $\mathrm{W}, \mathrm{Zheng} \mathrm{Z}$, et al. Mmu_circ_003795 regulates osteoblast differentiation and mineralization in MC3T3-E1 and MDPC23 by targeting COL15A1. Mol
Jiang et al. A new study of infrapatellar fat pad/synovium unit

Med Rep 2020;22:1737-46.

43. Yang XM, Song YQ, Li L, et al. miR-1249-5p regulates the osteogenic differentiation of ADSCs by targeting PDX1. J Orthop Surg Res 2021;16:10.

44. Nakaoka A, Nakahana M, Inubushi S, et al. Exosomemediated radiosensitizing effect on neighboring cancer cells via increase in intracellular levels of reactive oxygen species. Oncol Rep 2021;45:13.

45. Wang T, Xu Y, Liu X, et al. miR-96-5p is the tumor suppressor in osteosarcoma via targeting SYK. Biochem Biophys Res Commun 2021;572:49-56.

46. Lulla AR, Slifker MJ, Zhou Y, et al. miR-6883 Family miRNAs Target CDK4/6 to Induce G1 Phase Cell-Cycle Arrest in Colon Cancer Cells. Cancer Res 2017;77:6902-13.

(English Language Editor: C. Betlzar)

Cite this article as: Jiang T, Lu Y, Chen Z, Lin X, Zhang J, Shan J, Lu C, Zhao C, Xu X, Liu W. RNA sequencing reveals the circular RNA expression profiles of the infrapatellar fat pad/synovium unit. Ann Transl Med 2021;9(22):1685. doi: 10.21037/atm-21-5739 